\title{
Moving beyond isolated events
}

\author{
Research addressing compound and connected events, and their integrated risk to the natural and built world, \\ is gaining momentum. Paradigms are now evolving to classify and analyse the processes forming such links - \\ whether physical or societal, direct or indirect - and the role of climate change in their ultimate impacts.
}

T he headlines in May and June of tropical cyclones making landfall have been sobering reminders that climate extremes and their impacts continue despite the pandemic lockdowns. In May, Cyclone Vongfong crossed the Philippines while Amphan devastated eastern India and Bangladesh, both in the midst of the global COVID-19 crisis. Cyclone Nisarga made landfall along the coast of western India two weeks later.

Not only do these storms have compound extremes like storm surge, high winds and heavy rainfall, but they can damage infrastructure and hinder disaster response protocols, leading to societally connected events that further stress an already vulnerable population. In the build-up to Amphan's landfall, evacuation centres were housing the emergency response to COVID19 , and physical distancing policies meant they could not be filled to capacity ${ }^{1,2}$. In a Comment in this issue, Carly Phillips and co-authors discuss the compound risks that have resulted from COVID-19 and those expected over the coming months, highlighting the need to rewrite emergency response strategies to consider public health and climate system interactions that have been unfamiliar until now.

Understanding the vulnerability of the natural and built environment to linked events is a fast-growing discipline ${ }^{3-6}$. Colin Raymond and colleagues offer a Perspective describing connected extreme events, using 'connected' to convey a societal element linking extreme events rather than physical drivers alone. The authors exemplify connected extremes with Hurricanes Harvey and Maria in 2017. These were unrelated storms nearly a month apart, but their sequence, combined with a poor emergency response, led to disproportionate impacts. Harvey flooded parts of Texas, USA, necessitating aid from the US Federal Emergency Management Agency (FEMA). Weeks later, when Maria struck Puerto Rico, FEMA's resources were depleted, and the island's vulnerability - already high from poor infrastructure and an older population - was further exacerbated.

Raymond and colleagues argue that deep cross-sectoral and interdisciplinary collaboration is necessary to prepare for and respond to such connected extremes.

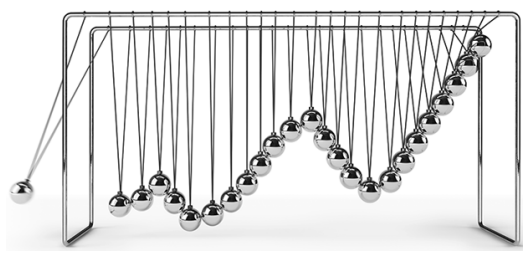

Credit: Atomic Imagery / DigitalVision / Getty

Impacts depend on the politics, economy, infrastructure and cultural networks within and among countries, which themselves rely on shifting societal mechanisms that can increase or decrease resilience or vulnerability. This holds true across all major sectors, including food ${ }^{7}$ and infrastructure ${ }^{8}$. However, it is an overwhelming task to combine physical modelling with society's behaviour during such events; their rareness makes them unfit for a one-size-fits-all approach. For researchers, it will be important to focus on which societal systems fail under such circumstances, why this happens and who is ultimately impacted.

In a companion Review ${ }^{9}$ in Nature Reviews Earth \& Environment, Jakob Zscheischler and co-authors summarize the state of the science on compound events, which focus on physical rather than societal interactions and are characterized as having modulators, drivers, hazards and impacts. The authors classify compound events into preconditioned, multivariate, temporally and spatially compounding types, though these boundaries can blur. Importantly, each of these characteristics can be affected by climate change.

Quantifying compound and connected events is inevitably a numbers game. Climate and societal extremes already live in the tails of probability distributions where few observations exist, and estimating their relationships and co-occurrence will require innovative methods. Statistical and modelling approaches exist, though techniques that can characterize interactions and feedbacks between society and the physical world are less quantitative and rely on sectoral expertise, interaction with policy- and decision-makers, and input from stakeholders and practitioners. A common thread in the linked events literature is the need for more of these interactions among private, public and academic sectors.
Another consideration is that not all risks compound or reinforce one another. In a Comment, John Hillier and colleagues discuss the need to account for mutual exclusivity in multi-hazard risk assessment. As an example, they consider the impact of the El Niño/ Southern Oscillation (ENSO) on Australia. In the summertime, an active El Niño decreases the number of landfalling tropical cyclones yet increases drought likelihood and wildfire risk, whereas the La Niña phase has the opposite effect. Thus, no matter the ENSO phase, simultaneous cyclone and drought impacts are unlikely. Hillier and co-authors argue that the majority of the compound events literature focuses on dependencies that exacerbate rather than decrease multi-hazard risk in high-impact situations. But both are important, and a more expansive definition of compound risk is suggested that accounts for both an increase or a decrease in risk relating to severe scenarios.

Establishing typologies for linked events, and instating more rigorous methods around them, is important. Together, these Comment and Review articles offer pathways to progress research that quantifies the ultimate likelihood, risk and impact of such phenomena. However, while distinctions between these concepts continue to crystallize, it is important to remember what links them: a single event may have a specific impact by itself, but when combined with additional physical or societal forces, the associated risks and hazards can interact nonlinearly.

Published online: 30 June 2020

https://doi.org/10.1038/s41558-020-0846-5

References

1. Amphan: Kolkata devastated as cyclone kills scores in India and Bangladesh. BBC News (21 May 2020); https://bbc.in/3fHma5s

2. Khadka, N. S. Social distancing: when extreme weather and coronavirus collide. BBC News (20 May 2020); https://bbc. in/3hWfIJB

3. Seneviratne, S. I. et al. in IPCC Managing the Risks of Extreme Events and Disasters to Advance Climate Change Adaptation (eds Field C. B. et al.) 109-230 (Cambridge Univ. Press, 2012).

4. Leonard, M. et al. WIREs Clim. Change 5, 113-128 (2014).

5. Zscheischler, J. \& Seneviratne, S. I. Sci. Adv. 3, el700263 (2017).

6. Zscheischler, J. et al. Nat. Clim. Change 8, 469-477 (2018).

7. Gaupp, F. et al. Nat. Clim. Change 10, 54-57 (2020).

8. Moftakhari, H. \& AghaKouchak, A. Environ. Res. Lett. 14, 10 (2019).

9. Zscheischler, J. Nat. Rev. Earth Environ. https://doi.org/10.1038/ s43017-020-0060-z (2020). 